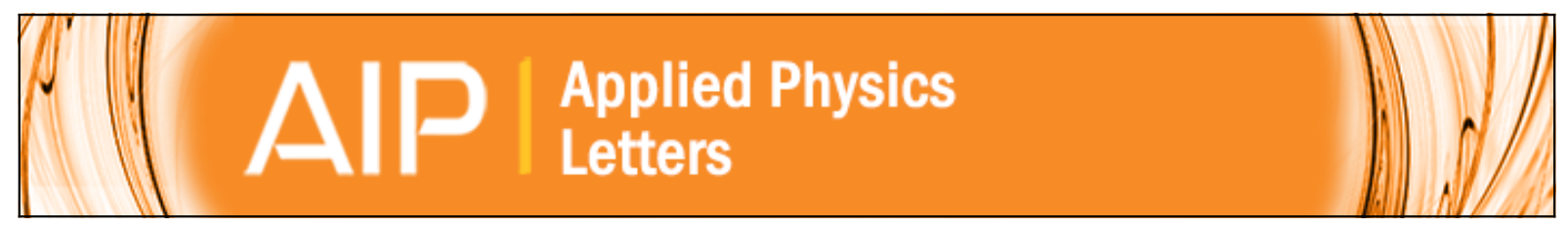

\title{
Electroinductive waves in chains of complementary metamaterial elements
}

M. Beruete, F. Falcone, M. J. Freire, R. Marqués, and J. D. Baena

Citation: Applied Physics Letters 88, 083503 (2006); doi: 10.1063/1.2176850

View online: http://dx.doi.org/10.1063/1.2176850

View Table of Contents: http://scitation.aip.org/content/aip/journal/apl/88/8?ver=pdfcov

Published by the AIP Publishing

\section{Articles you may be interested in}

Theoretical and experimental study of the backward-wave radiation using resonant-type metamaterial transmission lines

J. Appl. Phys. 112, 104513 (2012); 10.1063/1.4766310

Mapping inter-element coupling in metamaterials: Scaling down to infrared

J. Appl. Phys. 111, 094904 (2012); 10.1063/1.4711092

Left handed transmission properties of planar metamaterials based on complementary double-ring resonators

J. Appl. Phys. 108, 033717 (2010); 10.1063/1.3437087

The radiation from a planar metamaterial waveguide

J. Appl. Phys. 107, 113301 (2010); 10.1063/1.3428479

Stacked complementary metasurfaces for ultraslow microwave metamaterials

Appl. Phys. Lett. 96, 164103 (2010); 10.1063/1.3413958

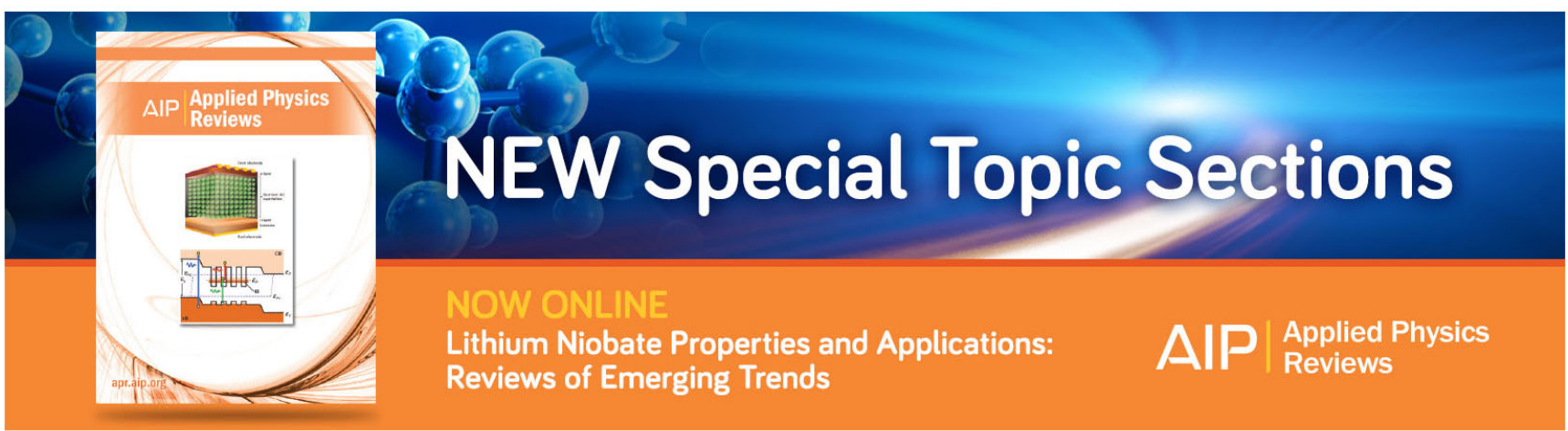




\title{
Electroinductive waves in chains of complementary metamaterial elements
}

\author{
M. Beruete and F. Falcone \\ Departamento de Ingeniería Eléctrica y Electrónica, Universidad Pública de Navarra, \\ Campus Arrosadía s/n, E 31006 Pamplona, Spain \\ M. J. Freire, ${ }^{\text {a) }}$ R. Marqués, and J. D. Baena \\ Departamento de Electrónica y Electromagnetismo, Facultad de Física, Universidad de Sevilla, \\ Avda. Reina Mercedes s/n, E 41012 Seville, Spain
}

(Received 22 July 2005; accepted 4 January 2006; published online 22 February 2006)

\begin{abstract}
Electronductive waves supported by chains of resonators drilled on a metallic plate are presented. Propagation of energy comes as a consequence of the electric coupling between these resonators. Therefore, these waves are termed as electroinductive waves. They can be interpreted as the dual counterpart of the so-called magnetoinductive waves, which are due to the mutual inductances along chains of resonators. In order to show their existence, some electromagnetic simulations and experiments have been carried out, using as resonators the complementary particle of the split ring resonator. The reported result opens the way to a high variety of applications in one- and two-dimensional devices, such as transducers, delay lines, bends, power dividers, couplers, antennas, lenses, etc. (C) 2006 American Institute of Physics. [DOI: 10.1063/1.2176850]
\end{abstract}

After the seminal paper of Pendry ${ }^{1}$ where split ring resonators (SRRs) were proposed and applied to the design of artificial media with negative permeability, many researchers became interested in the properties of these particles. Apart from the obvious applications in designing materials with exotic properties, such as negative refractive index and evanescent wave focusing, other proposals concentrated on the guiding properties of these particles. Recently, Shamonina et al. analyzed both theoretically ${ }^{2-4}$ and experimentally ${ }^{5}$ the properties of a new kind of wave supported by arrays of capacitively loaded rings, the magnetoinductive (MI) waves. These waves owe their existence to the magnetic coupling between adjacent resonators, so they can exist in any periodic structure with magnetic coupling between elements. In the aforementioned series of papers, Shamonina et al. exhaustively described the properties of MI waves (MIWs) in one, two, and three dimensions, as well as the effects of unmatched loadings, bends, couplings, losses in conductors, tolerances in fabrication, etc., and compared the characteristics of MI waveguides with standard transmission lines. Soon after, some of the authors explored the transduction from electromagnetic waves to MIWs in conventional planar circuits, ${ }^{6}$ as well as the usefulness of MIWs in the design of planar superlenses. ${ }^{7}$

Propagation of electromagnetic signals by chains of electrically coupled metallic particles is an effect known since far at microwave frequencies, ${ }^{8}$ and more recently observed at optical frequencies. ${ }^{9,10}$ In this letter, we present the basic concepts of a kind of wave supported by chains of planar resonators drilled on a metallic plate and due to the electric coupling between them. These waves are referred as electroinductive waves (EIWs), from its parallelism (or duality) with the aforementioned MIWs, studied in Refs. 2-7. To begin with, we will consider the EIWs guided by a chain of complementary SRRs (CSRRs). This resonator is the dual counterpart of the SRR [see Fig. 1(a)]. It was recently proposed by some of the authors in order to provide planar

${ }^{a)}$ Electronic mail: freire@us.es structures with a strong negative electric polarizability. ${ }^{11}$ Therefore, its usefulness for the guidance of EIWs is apparent. CSRRs can be analyzed in their first resonance by means of an LC circuit, dual in many aspects to that commonly used for the analysis of SRRs. ${ }^{12}$ When a chain of CSRRs is formed by arraying particles, the circuit model reported in Ref. 12 should be modified in order to include the mutual capacitance, $C_{M}$, between adjacent resonators. Furthermore, the capacitance between the central disk of the CSRR and the ground should be modified since this ground is partly reduced by the presence of the nearest neighbors in the chain. Therefore, as a first approximation, $C_{C} \approx 2 C_{M}+C_{G}$, where $C_{C}$ is the CSRR capacitance defined in Ref. 12, and $C_{G}$ is the capacitance to ground in the chain of CSRRs. Once

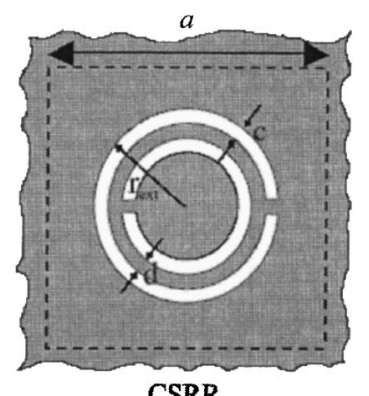

CSRR

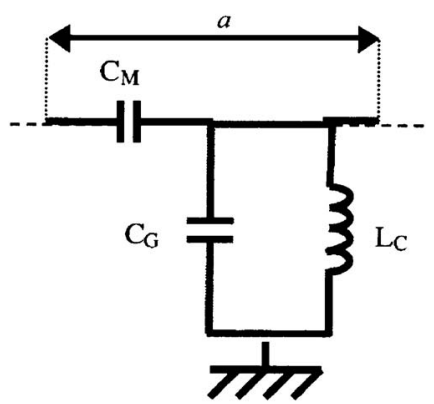

FIG. 1. (a) CSRR topology. (b) Equivalent circuit for the unit cell of an infinite chain of LC resonators. Electric coupling between resonators is represented by a mutual capacitance $C_{M}$. 
these modifications have been introduced in the circuit model of the CSRR, the equivalent circuit shown in Fig. 1(b) arises for the unit cell of the chain of CSRRs, where $L_{C}$ is the CSRR inductance, as it is defined in Ref. 12 and $a$ is the period of the chain. This equivalent circuit can be used for the determination of the dispersion relation for EIWs in the nearest-neighbor approximation, which is enough to obtain the salient features in this kind of one-dimensional system. ${ }^{3,6}$ After some algebra, this dispersion relation is found to be

$$
\frac{\omega^{2}}{\omega_{0}^{2}}=1+2 \omega^{2} C_{M} L_{C} \cos (k a)
$$

where

$$
\omega_{0}^{2}=\frac{1}{\left(C_{G}+2 C_{M}\right) L_{C}} \approx \frac{1}{C_{C} L_{C}} .
$$

The aforementioned duality between EIWs and MIWs clearly arises, after some algebra, from these equations. In fact, if the frequency bandwidth of Eq. (1) is assumed to be small, we can take $\omega \approx \omega_{0}$ in the right-hand side of Eq. (1), which leads to

$$
\frac{\omega^{2}}{\omega_{0}^{2}} \approx 1+\frac{2 C_{M}}{C_{G}+2 C_{M}} \cos (k a) \approx 1+\frac{2 C_{M}}{C_{C}} \cos (k a) .
$$

This expression can be now compared with the dispersion relation for MIWs in a chain of SRRs (Ref. 6) which, in the same approximation, can be written as

$$
\frac{\omega^{2}}{\omega_{0}^{2}}=\left[1+\frac{2 M}{L} \cos (k a)\right]^{-1} \approx 1-\frac{2 M}{L} \cos (k a),
$$

where $M$ and $L$ are the mutual inductance and the selfinductance, respectively, of the SRRs. ${ }^{6}$ Both relations (1) and (4) coincide if the resonant frequency of the CSRRs and the SRRs is the same and we change $C_{M}$ by $K M$ and $C_{C}$ by $K L$, where $K$ is an arbitrary constant. If the CSRRs and the SRRs are embedded in an homogeneous isotropic medium, these conditions are fulfilled with $K=4(\varepsilon / \mu) .{ }^{12}$ Similar relationsalthough with different values of $K$-arise when both elements are etched on the plane interface between two semiinfinite media. ${ }^{12}$ For intermediate situations, this equivalence will be only approximated. In summary, EIWs can be considered as the dual counterparts of MIWs, having a similar electromagnetic behavior (in the sense of Babinet's principle. $^{11}$ )

In order to gain more insight into the details of EIWs propagation, several simulations using the commercial software CST microwave studio ${ }^{\mathrm{TM}}$ have been carried out. The simulated structure consists of an electric monopole over a lossless infinite ground plate where a linear CSRR array has been drilled in (see Fig. 2). As is readily seen, near the resonance this chain of CSRRs supports a traveling wave, thus allowing for the guidance of the electromagnetic energy. In Fig. 2(a), the density of electric energy for a long row of 50 coupled CSRRs is shown. It is clear that a guided wave exists along the chain, and that electric energy is trapped in the vicinity of the particles being negligible out of them. It is worth noting that the standing wave pattern along the chain of resonators, due to the mismatch introduced at the end of the chain. For comparison purposes, the density of energy of a single CSRR located at the same distance as the last one of the chain is shown in Fig. 2(b). Note that the source is unable
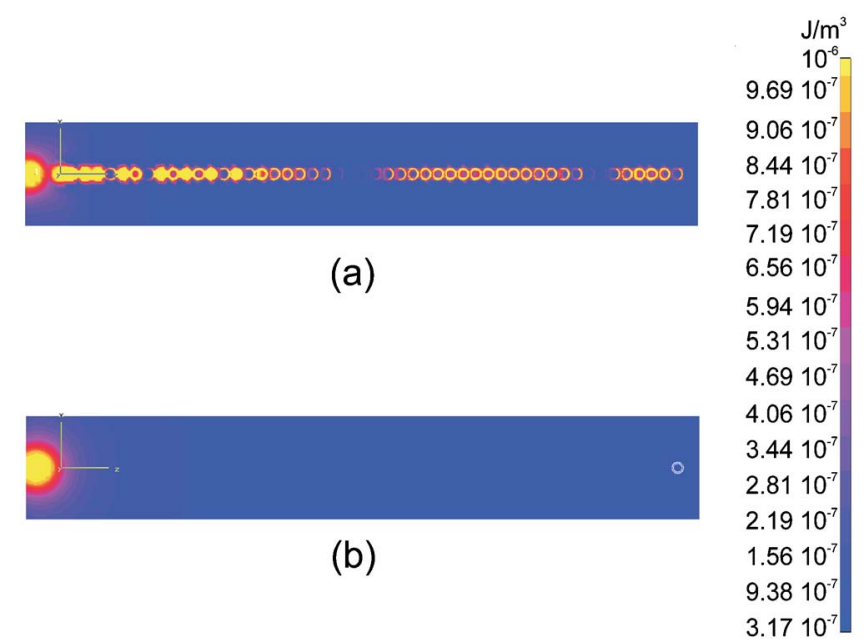

FIG. 2. (Color online) (a) Electric energy density created by a monopole (on the left) on a perfect conductor ground plane drilled by a chain of $50 \mathrm{CS}$ RRs. The parameters of the resonators are (as shown in Fig. 1): $r_{\text {ext }}$ $=3.5 \mathrm{~mm}, c=d=0.4 \mathrm{~mm}, a=8 \mathrm{~mm}$. (b) A single resonator is located on the right at a distance equal to $400 \mathrm{~mm}$, i.e., the distance of the last resonator of the aforementioned chain.

to excite the ring by itself, which clearly states for a transport of energy when resonators are put together.

Once the existence of the EIW has been shown, further steps toward a more practical prototype were made. Since many of the present microwave devices are fabricated in planar technology, it is interesting to show the application of EIWs in microstrip technology. For this analysis, both simulations and experiments have been carried out. The analyzed structure is shown in Fig. 3(a). This structure was fabricated and measured. It consists of a chain of five resonators which were etched on the ground plane of a microwave circuit board. Two microstrip lines lay over the resonators, thus forming a transducer between the electromagnetic waves propagating along the microstrips and the EIWs. The resonators are essentially the complementary ones of the split squared ring resonator (CSSRR) previously used in Ref. 6. The only difference is that the slots of the CSSRRs have been enlarged by comparison with the arms of the SSRR in Ref. 6. It has been found that this modification contributes to a reduction in the ohmic losses in the resonator. The CSSRRs at the beginning and at the end of the chain were placed just below the open end of the microstrip lines, where the electric field is maximum, in order to enhance the electric coupling between the microstrip and the CSSRR chain (this is a key difference between the EIW and the MIW transducer reported in Ref. 6 , where the resonators should be placed at a distance $\lambda / 4$ from the microstrip open end, in order to enhance the magnetic coupling). Figure 3(b) shows both the simulation and the measurement of the transmission coefficient versus frequency for the structure sown in Fig. 3(a). The simulations were obtained with the commercial software ENSEMBLE ${ }^{\mathrm{TM}}$ and the measurements were carried out with a Vector Network Analyzer HP 8510 B. The simulation for the lossless case (solid gray curve) shows a passband near the resonance of the individual CSSRRs; well defined and with small ripples. When losses are taken into account in the simulation (solid black curve), the shape of the passband remains basically the same as for the lossless case, but with some attenuation. The experimental results are shown by black markers. As can be seen, the experimental response is 

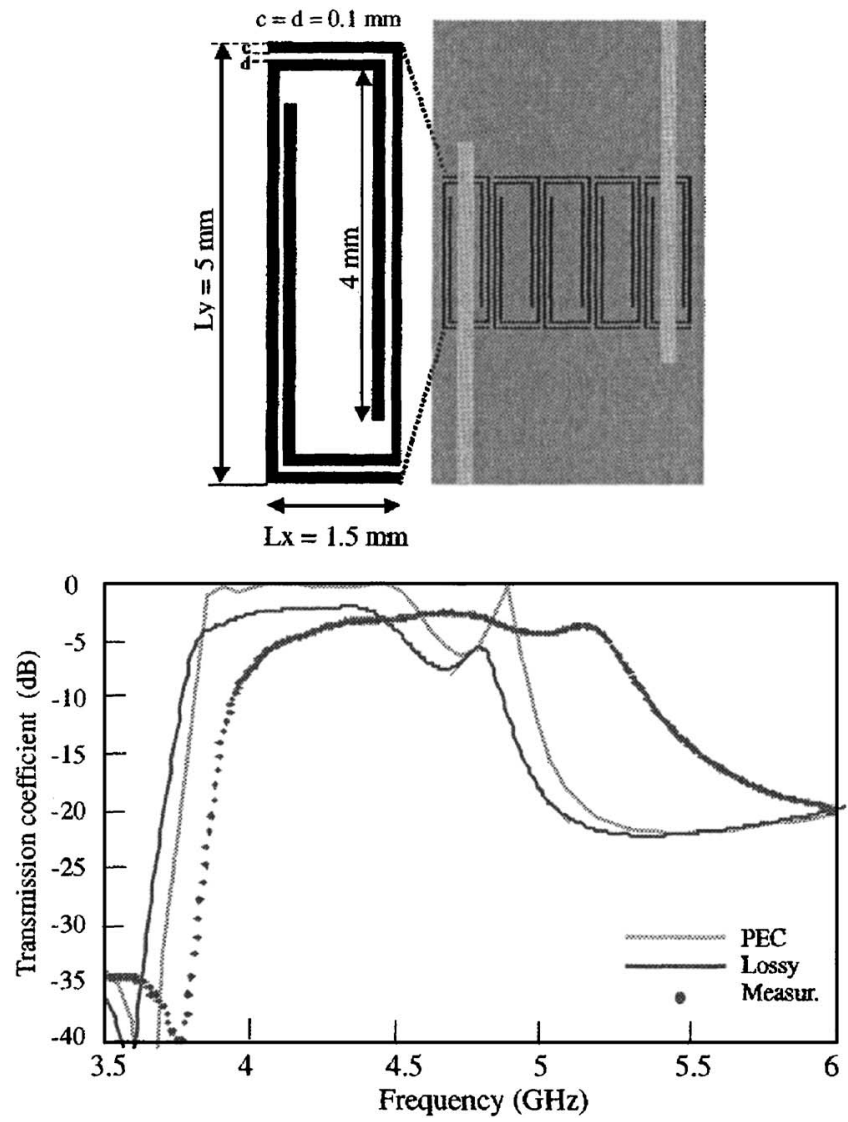

FIG. 3. (a) Sketch of the CSSRR transducer in microstrip technology. The parameters of the resonators are: $L x=1.5 \mathrm{~mm}, L y=5 \mathrm{~mm}, c=d=0.1 \mathrm{~mm}$. The periodicity is $a=1.6 \mathrm{~mm}$. The substrate thickness was $0.635 \mathrm{~mm}$ and the dielectric permittivity $\varepsilon_{r}=10$. The microstrip lines were designed to have $50 \Omega$ of characteristic impedance. (b) Simulated transmission response for the lossless case or perfect conductor metallization (gray), simulated transmission for the lossy or copper $\left(\sigma=5.8 \times 10^{7} \mathrm{~S} / \mathrm{m}\right)$ metallization (black) and measured transmission for the latter case (black marker).

similar to that given by the simulations but it is shifted in frequency, probably due to tolerances in the fabrication process. The results in Fig. 3 show that the proposed structure behaves in a similar way as the MIW transducer reported in
Ref. 6, thus confirming the duality between EIWs and MIWs.

In summary, the existence of EIWs in chains of electrically coupled CSRRs has been demonstrated both theoretically and experimentally. The duality between EIWs and previously reported MIWs has been discussed, and the ability of long CSRR chains to transport electromagnetic energy along many periods has been shown. A practical transducer between electromagnetic and EIWs in planar technology has been proposed and analyzed. Regarding practical applications, EIWs can be an alternative to MIWs for the guidance of electromagnetic energy, as well as for the design of couplers, delay lines and other planar devices, when electric couplings rather than magnetic couplings are imposed, or simply desired for the design.

This work has been supported by DGI, Ministerio de Educación y Ciencia (SPAIN), under project Contract Nos. TIC2002-04528-C02-01 and TEC2004-04249-C02-02. The authors thank their laboratory assistant Esperanza Rubio for her collaboration in the fabrication of the microwave circuits of this work.

${ }^{1}$ J. B. Pendry, A. J. Holden, D. J. Robbins, and W. J. Stewart, IEEE Trans. Microwave Theory Tech. 47, 2075 (1999).

${ }^{2}$ E. Shamonina, V. A. Kalinin, K. H. Ringhofer, and L. Solymar, Electron. Lett. 38, 371 (2002).

${ }^{3}$ E. Shamonina, V. A. Kalinin, K. H. Ringhofer, and L. Solymar, J. Appl. Phys. 92, 6252 (2002).

${ }^{4}$ E. Shamonina and L. Solymar, J. Phys. D 37, 362 (2004).

${ }^{5}$ M. C. K. Wiltshire, E. Shamonina, I. R. Young, and L. Solymar, Electron. Lett. 39, 215 (2003).

${ }^{6}$ M. J. Freire, R. Marqués, F. Medina, M. A. G. Laso, and F. Martín, Appl. Phys. Lett. 85, 4439 (2004).

${ }^{7}$ M. J. Freire and R. Marqués, Appl. Phys. Lett. 86, 182505 (2005).

${ }^{8}$ J. Shefer, IEEE Trans. Microwave Theory Tech. 11, 55 (1963).

${ }^{9}$ M. L. Brongersma, J. W. Hartmann, and H. A. Artwater, Phys. Rev. B 62, R16356 (2000).

${ }^{10}$ W. H. Weber and G. W. Ford, Phys. Rev. B 70, 125429 (2004).

${ }^{11}$ F. Falcone, T. Lopetegi, M. A. G. Laso, J. D. Baena, J. Bonache, M. Beruete, R. Marques, F. Martin, and M. Sorolla, Phys. Rev. Lett. 93, 197401 (2004).

${ }^{12}$ J. D. Baena, J. Bonache, F. Martín, R. Marqués, F. Falcone, T. Lopetegi, M. A. G. Laso, J. García, I. Gil, M. Flores, and M. Sorolla, IEEE Trans. Microwave Theory Tech. 53, 1451 (2005). 